\title{
Secondary Degeneration Detected by Combining Voxel-Based Morphometry and Tract-Based Spatial Statistics in Subcortical Strokes with Different Outcomes in Hand Function
}

D. Yin, X. Yan, M. Fan, Y. Hu, W. Men, L. Sun, and F. Song

O-

\begin{abstract}
BACKGROUND AND PURPOSE: Secondary degeneration of the pyramidal tract after focal motor pathway stroke has been observed by diffusion tensor imaging. However, the relationships between outcomes in hand function and secondary degeneration in widespread regions are not well understood. For the first time, we investigated the differences of secondary degeneration across the whole brain between subgroups of patients with stroke.
\end{abstract}

MATERIALS AND METHODS: We selected 23 patients who had a subcortical stroke in the left motor pathway and displayed only motor deficits. The patients were divided into 2 subgroups: $\mathrm{CPH}$ (11 patients) and PPH (12 patients). Twelve healthy controls matched for age and handedness were also recruited. We used both optimized VBM and TBSS to explore differences of FA across the whole brain between CPH and PPH. Furthermore, ROI analysis was carried out in the identified regions detected by VBM analysis to further quantify the degree of secondary degeneration in the $\mathrm{CPH}$ and $\mathrm{PPH}$ and compare these with healthy controls.

RESULTS: Compared with PPH, FA was significantly decreased in the CPH in widespread regions of the motor system remote from the primary lesion, including the ipsilesional brain stem, medial frontal gyrus, precentral gyrus, superior temporal gyrus, supplementary motor area, and contralesional postcentral gyrus. In addition, FA within these identified regions correlated with Fugl-Meyer Assessment scores (hand+ wrist).

CONCLUSIONS: This study suggests a potential biomarker for outcome differences in hand function after subcortical stroke.

ABBREVIATIONS: $\quad \mathrm{CPH}=$ completely paralyzed hands; $\mathrm{FA}=$ fractional anisotropy; $\mathrm{PPH}=$ partially paralyzed hands; TBSS $=$ tract-based spatial statistics; $\mathrm{VBM}=$ voxel-based morphometry

S econdary degeneration of the pyramidal tract after focal motor pathway stroke has been detected by DTI. A strong correlation between degeneration in the cerebral peduncle and functional outcome of the upper limbs has been detected in patients with chronic stage stroke. ${ }^{1}$ Longitudinal studies have also demonstrated a progressive decrease of FA along the pyramidal tract in

Received July 28, 2012; accepted after revision October 8.

From the Shanghai Key Laboratory of Magnetic Resonance (D.Y., X.Y., M.F., W.M.), Department of Physics, East China Normal University, Shanghai, China; Department of Rehabilitation Medicine (Y.H., L.S., F.S.), Huashan Hospital, Fudan University, Shanghai, China; and State Key Laboratory of Medical Neurobiology (Y.H.), Fudan University, Shanghai, China.

This study was supported by the Excellent Young Teachers Program of Shanghai Medical College of Fudan University (Grant No. 124).

Please address correspondence to Fan Song, Department of Rehabilitation Medicine, Huashan Hospital, Fudan University, 12 Wulumuqi Middle Rd, Shanghai 200040, China; e-mail: songfan@medmail.com.cn; or Mingxia Fan, Shanghai Key Laboratory of Magnetic Resonance, East China Normal University, 3663 North Zhong-Shan Rd, Shanghai 200062, China; e-mail: mxfan@phy.ecnu.edu.cn

- Indicates open access to non-subscribers at www.ajnr.org

7 Indicates article with supplemental on-line figures.

http://dx.doi.org/10.3174/ajnr.A3410 the cerebral peduncle below the primary lesion. ${ }^{2,3}$ However, these studies have mainly focused on descending degeneration at the level of the cerebral peduncle, and ROI or segment-of-corticospinal-tract analyses were often carried out based on a priori hypotheses, ${ }^{4,5}$ which are time consuming and do not easily allow for comparisons of many brain regions or large subject groups. Therefore, secondary degeneration in more widespread regions of the motor system has rarely been studied.

$\mathrm{VBM}^{6}$ is commonly used in whole-brain analyses to localize abnormalities in the presence of pathologic changes. Usually, brain images from different subjects are transformed to a standard space by registering them to a template. Thus, intersubject voxelwise comparisons across the whole brain can be performed by use of the unified coordinate system. Because the frequently used nomalization techniques (eg, performing in SPM or FSL) are impossible to achieve a perfect match, smoothing is subsequently applied to reduce the individual variability and misalignments. However, smoothing may increase the partial volume effects. In contrast, TBSS $^{7}$ is an automated, observer-independent approach for assessing group-wise microstructural differences in the major 
Table 1: Clinical and demographic data of 23 patients with subcortical stroke

\begin{tabular}{|c|c|c|c|c|c|c|}
\hline Pt No. & Sex & Age (y) & $\begin{array}{l}\text { Location of } \\
\text { Lesion }\end{array}$ & $\begin{array}{c}\text { Time after Stroke } \\
\text { (months) }\end{array}$ & $\begin{array}{l}\text { Lesion Volume } \\
(\mathrm{mL})\end{array}$ & $\begin{array}{l}\text { FMA Scores } \\
\text { (hand+wrist) }\end{array}$ \\
\hline \multicolumn{7}{|c|}{ PPH Group } \\
\hline 01 & $M$ & 60 & $\mathrm{~L}, \mathrm{IC}, \mathrm{Th}^{\mathrm{a}}$ & 53 & 35.32 & 12 \\
\hline 02 & $\mathrm{~F}$ & 48 & $\mathrm{~L}, \mathrm{IC}, \mathrm{BG}$ & 23 & 9.38 & 6 \\
\hline 03 & $M$ & 76 & $\mathrm{~L}, \mathrm{IC}$ & 21 & 62.18 & 22 \\
\hline 04 & $M$ & 60 & $\mathrm{~L}, \mathrm{IC}, \mathrm{BG}$ & 36 & 16.48 & 6 \\
\hline 05 & $M$ & 71 & L, IC, $\mathrm{Th}^{\mathrm{a}}$ & 22 & 17.64 & 11 \\
\hline 06 & $M$ & 63 & $\mathrm{~L}, \mathrm{IC}, \mathrm{BG}, \mathrm{Th}$ & 3 & 22.14 & 13 \\
\hline 07 & $M$ & 54 & $\mathrm{~L}, \mathrm{IC}, \mathrm{Th}^{\mathrm{a}}$ & 3 & 12.35 & 23 \\
\hline 08 & $M$ & 60 & $\mathrm{~L}, \mathrm{IC}, \mathrm{Th}^{\mathrm{a}}$ & 11 & 26.96 & 23 \\
\hline 09 & $M$ & 65 & L, IC, Th & 12 & 8.85 & 23 \\
\hline 10 & $M$ & 53 & $\mathrm{~L}, \mathrm{IC}, \mathrm{BG}, \mathrm{Th}^{\mathrm{a}}$ & 22 & 125.10 & 15 \\
\hline 11 & $M$ & 65 & $\mathrm{~L}, \mathrm{IC}, \mathrm{BG}^{\mathrm{a}}$ & 6 & 81.78 & 20 \\
\hline 12 & $M$ & 56 & $\mathrm{~L}, \mathrm{IC}$ & 7 & 10.55 & 11 \\
\hline \multicolumn{7}{|c|}{ CPH Group } \\
\hline 01 & $M$ & 67 & L, IC & 17 & 12.33 & 2 \\
\hline 02 & $M$ & 57 & L, IC, Th & 19 & 23.73 & 0 \\
\hline 03 & $\mathrm{~F}$ & 75 & $\mathrm{~L}, \mathrm{IC}, \mathrm{CR}$ & 24 & 7.80 & 4 \\
\hline 04 & $M$ & 63 & $\mathrm{~L}, \mathrm{BG}, \mathrm{IC}, \mathrm{Th}^{\mathrm{a}}$ & 16 & 61.52 & 1 \\
\hline 05 & $\mathrm{~F}$ & 65 & L, IC, Th & 17 & 29.59 & 1 \\
\hline 06 & $\mathrm{~F}$ & 68 & $\mathrm{~L}, \mathrm{BG}, \mathrm{IC}, \mathrm{Th}^{\mathrm{a}}$ & 62 & 25.51 & 0 \\
\hline 07 & $M$ & 68 & $\mathrm{~L}, \mathrm{IC}, \mathrm{Th}^{\mathrm{a}}$ & 47 & 32.73 & 1 \\
\hline 08 & $M$ & 62 & L, BG, IC, $T h^{\mathrm{a}}$ & 12 & 156.14 & 0 \\
\hline 09 & $M$ & 53 & $\mathrm{~L}, \mathrm{BG}, \mathrm{IC}, \mathrm{Th}^{\mathrm{a}}$ & 86 & 55.53 & 1 \\
\hline 10 & $\mathrm{~F}$ & 50 & $\mathrm{~L}, \mathrm{BG}, \mathrm{IC}, \mathrm{Th}^{\mathrm{a}}$ & 13 & 18.66 & 0 \\
\hline 11 & $M$ & 62 & L, IC, Th & 6 & 19.16 & 4 \\
\hline
\end{tabular}

Note:-M indicates male; F, female; L, left; R, right; BG, basal ganglia; IC, internal capsule; Th, thalamus; CR, coronal radiata; FMA, Fugl-Meyer Assessment.

${ }^{a}$ The characteristics of the lesion are hemorrhagic; others are ischemic.

white matter pathways of the brain. It does not require smoothing and has more precise alignment. However, the result of TBSS cannot cover the regions of the whole brain, except on the main skeleton of white matter. Afzali et $\mathrm{al},{ }^{8}$ in a previous study assessing microstructure of white matter, used both VBM and TBSS to confirm their findings. In our study, we also used both VBM and TBSS as complementary methods to demonstrate abnormalities of the microstructure of white matter across the whole brain. We adopted an optimized strategy to register FA maps by using individual $b=0$ images (nondiffusion weighted), which are less affected by white matter degeneration compared with FA images.

In clinical observation, many patients with stroke seem to have similar location and extent of lesion but actually have prodigiously different outcomes in hand function. Some regain certain practical aspects of hand function, whereas some lose the practical function completely. The former are regarded as $\mathrm{PPH}$ and the latter as CPH. Changes in motor-related brain activation patterns might occur not as a function of time after stroke but as a function of motor recovery. ${ }^{9}$ Cerebral reorganization has been consistently demonstrated in patients with stroke by use of functional MR imaging, which depends on the integrity of the corticospinal system. ${ }^{10}$ However, regions where secondary degeneration contributes to differences of cerebral reorganization have not been completely identified. Moreover, the relationships between outcomes in hand function and secondary degeneration of the motor system are not clearly understood. We hypothesized that $\mathrm{CPH}$ and $\mathrm{PPH}$ have different degrees of secondary degeneration in widespread regions of the motor system and that the degree of secondary degeneration correlates with outcomes in hand function.

\section{MATERIALS AND METHODS \\ Participants}

From May 26, 2010 to November 28, 2011, a total of 23 patients with pure motor deficits from a subcortical stroke that occurred in the left motor pathway were selected from both outpatient and inpatient services at the Huashan Hospital affiliated with Fudan University. The patients were divided into 2 subgroups: $\mathrm{PPH}$ (12 patients, $11 \mathrm{men}$ ) and $\mathrm{CPH}$ (11 patients, 7 men). Twelve healthy controls ( 5 men) matched for age and handedness were also recruited from local communities. The Mini-Mental State Examination was used to evaluate the cognitive performance of patients with stroke, and a score of $>24$ on the Mini-Mental State Examination is regarded as having sufficient cognitive performance. ${ }^{11}$ Fugl-Meyer Assessment was performed to evaluate the motor functions of patients with stroke. ${ }^{12}$ To investigate hand function, we focused on the sum of Fugl-Meyer Assessment scores on the hand and wrist in our current study. Inclusion criteria were as follows: 1) all patients had first-onset stroke, 2) all participants were right-handed, 3) all participants had sufficient cognitive abilities (Mini-Mental State Examination >24), 4) at least 3 months from stroke onset was required for patients with $\mathrm{PPH}$ and 6 months for patients with $\mathrm{CPH}$ (because 6 months was identified as a prerequisite for $\mathrm{CPH}$ ), and 5) age was $45-80$ years. Exclusion criteria were as follows: 1) a contraindication to MR imaging, 2) quadriplegia, 3) a history of neurologic or psychiatric disorders, 4) diabetes, and 5) previous hand dysfunctions. Patient clinical characteristics and demographic data are summarized in Table 1, and the lesion with the maximal area in each patient is shown in Fig 1 . The protocol of this prospective study was approved by the Institutional Ethics Committee of East China Normal University, 


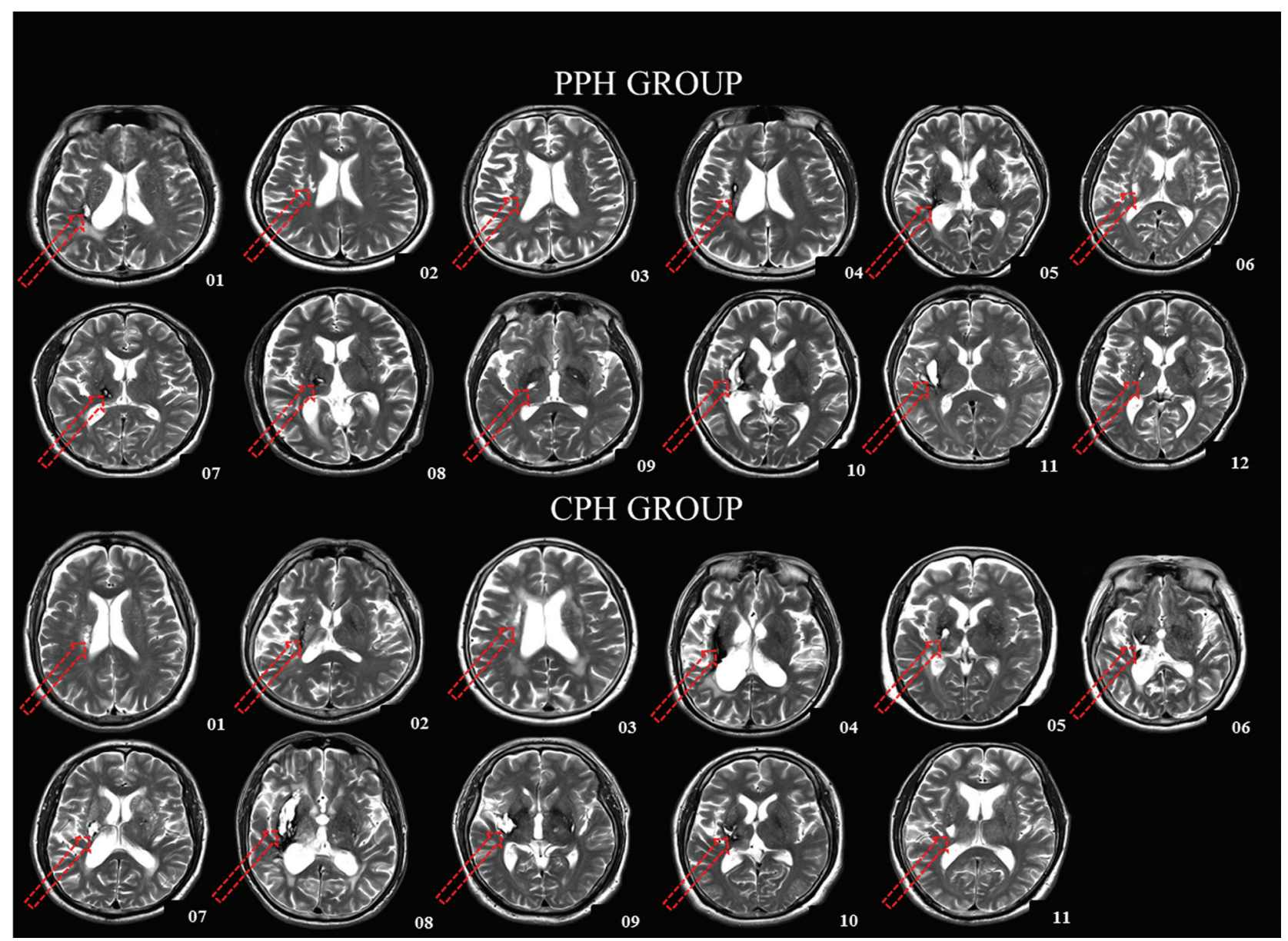

FIG 1. T2-weighted images show the largest lesion (red arrow) of each patient at the axial section. The locations and extent of the lesions are quite consistent across all of the patients. Arabic numerals denote the patient numbers.

Shanghai, China, and all participants or their guardians signed the informed consent forms.

We identified $\mathrm{CPH}$ and PPH by using the Paralyzed Hand Function Assessment, which involves 5 practical actions of the hand in daily life (On-line Fig. 1). We used this assessment to evaluate all of the patients. All those who could not complete any action were regarded as $\mathrm{CPH}$, and those who could complete at least 1 of the 5 actions were regarded as PPH. The assessment was performed by 2 experienced physicians from the Department of Rehabilitation Medicine, Huashan Hospital.

\section{Data Acquisition}

All images were acquired on a Trio 3T MR imaging scanner (Siemens, Erlangen, Germany) at the Shanghai Key Laboratory of Magnetic Resonance, East China Normal University. The protocol for DTI included the collection of 30 images with noncollinear diffusion gradients $\left(b=1000 \mathrm{~s} / \mathrm{mm}^{2}\right)$ and 1 non-diffusionweighted image $\left(b=0 \mathrm{~s} / \mathrm{mm}^{2}\right)$. Use of an integrated parallel acquisition technique with an acceleration factor of 2 reduced the acquisition time and the distortion from susceptibility artifacts. We used single-shot echo-planar imaging with detailed parameters as follows: number of axial sections, 40; section thickness, 3 $\mathrm{mm}$; gap, none; acquisition matrix, $128 \times 128$ (interpolation to
$256 \times 256$ ); TR, $6100 \mathrm{~ms}$; TE, $110 \mathrm{~ms}$; field of view, $256 \mathrm{~mm} \times 256$ $\mathrm{mm}$; and average, 2 . To identify the location and size of the lesion, we acquired $\mathrm{T} 2$-weighted images by using a turbo-spin-echo sequence: number of axial sections, 30; section thickness, $5 \mathrm{~mm}$; gap, none; TR, $6000 \mathrm{~ms}$; TE, $93 \mathrm{~ms}$; field of view, $220 \mathrm{~mm} \times 220$ $\mathrm{mm}$; flip angle, $120^{\circ}$; and acquisition matrix, $320 \times 320$.

\section{Data Preprocessing}

We preprocessed DTI data by using the FMRIB Software Library (FSL 4.1; http://www.fmrib.ox.ac.uk/fsl) by the following procedures: 1) all diffusion-weighted images were checked visually for apparent artifacts; 2) for each participant, diffusion-weighted images were registered to the corresponding $b=$ 0 images with an affine transformation to correct eddy-current distortion; 3) we obtained a binary brain mask of each participant from the respective $b=0$ images by using the Brain Extraction Tool; 4) by using FMRIB's diffusion tools, we calculated the diffusion tensor of each voxel within the brain mask by a linear least-square fitting algorithm, and after diagonalization of the diffusion tensor, we obtained 3 diffusion tensor eigenvalues $\left(\lambda_{1}, \lambda_{2}\right.$, and $\left.\lambda_{3}\right)$; and 5) FA maps were derived according to the following equation: 


$$
F A=\frac{\sqrt{\left(\lambda_{1}-\lambda_{2}\right)^{2}+\left(\lambda_{2}-\lambda_{3}\right)^{2}+\left(\lambda_{3}-\lambda_{1}\right)^{2}}}{\sqrt{2} \sqrt{\lambda_{1}{ }^{2}+\lambda_{2}{ }^{2}+\lambda_{3}^{2}}}
$$

\section{VBM Analysis of FA Maps between the CPH Group and the PPH Group}

In our current study, we performed VBM of FA maps by using statistical parametric mapping (SPM8, http://www.fil.ion. ucl.ac.uk/spm). However, registration plays a critical role in achieving precise VBM analysis, especially if registration is performed directly with FA maps, which may be affected by white matter degeneration caused by neurologic diseases or brain injury. To improve the accuracy of registration, we used an optimized VBM analysis for FA maps as follows. First, $b=0$ images of each $\mathrm{HC}$ were normalized to Montreal Neurological Institute space on the basis of an echo-planar imaging template, and normalized $b=0$ images of all healthy controls were averaged. Next, the averaged $b=0$ images were spatially smoothed by use of an isotropic Gaussian filter with a full width at half maximum of 6 $\mathrm{mm}$. Thus, we obtained a new template of $b=0$ image in the Montreal Neurological Institute space. Subsequently, individual $b=0$ images of all patients and healthy controls were registered to the new template (the normalization used the nonlinear registration method in SPM, including affine transformation and nonlinear transformation), and corresponding transformation matrices were also obtained. Then, FA maps of each participant were normalized to Montreal Neurological Institute space by using their corresponding transformation matrix and smoothed by using an isotropic Gaussian filter with a full width at half maximum of 6 $\mathrm{mm}$. Finally, a 2-sample $t$ test was performed for smoothed FA maps between $\mathrm{CPH}$ and $\mathrm{PPH}$. The threshold was set at a combined cutoff value of $P<.001$ (uncorrected) and a minimal cluster size of 20 voxels.

\section{TBSS Analysis of FA Maps between the CPH Group and the PPH Group}

We performed TBSS analysis by using FMRIB's free software FSL by the following procedures. ${ }^{7} 1$ ) After preprocessing, we first created a FA target image by averaging the normalized FA maps of all healthy controls in Montreal Neurological Institute space, which were obtained in the VBM analysis. Second, we estimated 2 transformations: nonlinear transformations from all individual FA maps to the FA target image and an affine transformation from the FA target image to a $1 \times 1 \times 1-\mathrm{mm}^{3}$ FA template in Montreal Neurological Institute 152 space, called FMRIB58_FA (the normalization used the nonlinear registration tool FNIRT in FSL). Finally, we combined the 2 transformations to take each original FA map directly into Montreal Neurological Institute 152 space. 2) We created the mean of all aligned FA images and applied "thinning" (nonmaximal suppression perpendicular to the local tract structure) to create a skeletonized mean FA image. The threshold was set at 0.2 to suppress areas of low mean FA and/or high intersubject variability. 3) We projected each participant's (aligned) FA image onto the skeleton by filling the skeleton with FA values from the nearest relevant tract center. This was achieved, for each skeleton voxel, by searching perpendicular to the local skeleton structure for the maximal value in the participant's FA image. 4) We carried out voxelwise statistics between $\mathrm{CPH}$ and $\mathrm{PPH}$ on the FA data in skeleton space. The threshold was set at $P<.001$ (uncorrected), and the statistical result was shown on the TBSS skeleton with the thickened image, which was performed by using the script of tbss_fill in FSL.

\section{ROI Analysis of FA Maps}

According to the significant differences between $\mathrm{CPH}$ and $\mathrm{PPH}$ FA maps detected by VBM analysis, we performed ROI analysis in the identified ROIs to further quantify the degree of secondary degeneration in $\mathrm{CPH}$ and $\mathrm{PPH}$ and to compare these with healthy controls. First, we made binary masks by using the clusters with significant group differences of FA maps between $\mathrm{CPH}$ and $\mathrm{PPH}$. Then, the respective binary masks were applied to the normalized FA maps of all participants ( $\mathrm{CPH}, \mathrm{PPH}$, and healthy controls). Finally, each ROI value for the patients was obtained by averaging all voxel values within each mask. In addition, correlation analysis was carried out between Fugl-Meyer Assessment scores (hand+wrist) and each ROI value across all patients. For ROI analysis, a $P$ value $<.05$ was considered to be statistically significant.

\section{RESULTS}

\section{Clinical Data}

No significant differences between the 2 subgroups were observed in age (PPH: mean $\pm \mathrm{SD}, 60.92 \pm 7.81$ years; $\mathrm{CPH}$ : mean $\pm \mathrm{SD}$, $62.73 \pm 7.21$ years; $P>.1$ ), time post stroke ( $\mathrm{PPH}$ : mean $\pm \mathrm{SD}$, $18.25 \pm 14.82$ months; $\mathrm{CPH}$ : mean \pm SD, $29.00 \pm 25.14$ months; $P>.1$ ), lesion volume ( $\mathrm{PPH}$ : mean $\pm \mathrm{SD}, 35.73 \pm 36.14 \mathrm{~mL}$; $\mathrm{CPH}$ : mean $\pm \mathrm{SD}, 40.24 \pm 41.87 \mathrm{~mL} ; P>.1)$, and Mini-Mental State Examination (PPH: mean $\pm \mathrm{SD}, 28.58 \pm 1.44$; $\mathrm{CPH}$ : mean $\pm \mathrm{SD}, 28.45 \pm 1.44 ; P>.1)$. We found a significant difference of the Fugl-Meyer Assessment score (hand+wrist) between the 2 subgroups (PPH: mean $\pm \mathrm{SD}, 15.42 \pm 6.54$; $\mathrm{CPH}$ : mean \pm $\mathrm{SD}, 1.27 \pm 1.49 ; P<.01)$. In addition, no significant correlation between lesion volume and Fugl-Meyer Assessment score (hand+wrist) was observed $(R=-0.13 ; P>.1)$.

\section{VBM Analysis of FA Maps between the CPH Group and PPH Group}

Compared with PPH, FA was significantly decreased in $\mathrm{CPH}$ in widespread regions of the motor system, including the ipsilesional medial frontal gyrus (cingulate gyrus), precentral gyrus, superior temporal gyrus, supplementary motor area, sublobar areas (internal capsule, thalamus, basal ganglia), brain stem, and the contralesional postcentral gyrus (Fig $2 A$ and Table 2).

\section{TBSS Analysis of FA Maps between the CPH Group and the PPH Group}

FA was significantly decreased in $\mathrm{CPH}$ compared with $\mathrm{PPH}$ in the ipsilesional medial frontal gyrus (cingulate gyrus), precentral gyrus, postcentral gyrus, superior temporal gyrus, internal capsule, brain stem, and contralesional precentral gyrus and postcentral gyrus (Fig $2 B$ ).

We observed that patterns of the FA difference between the $\mathrm{CPH}$ and $\mathrm{PPH}$ were similar between VBM analysis and TBSS analysis. The patterns consistently involved 4 parts of the pyramidal tract: ipsilesional cortical sensorimotor areas, perile- 


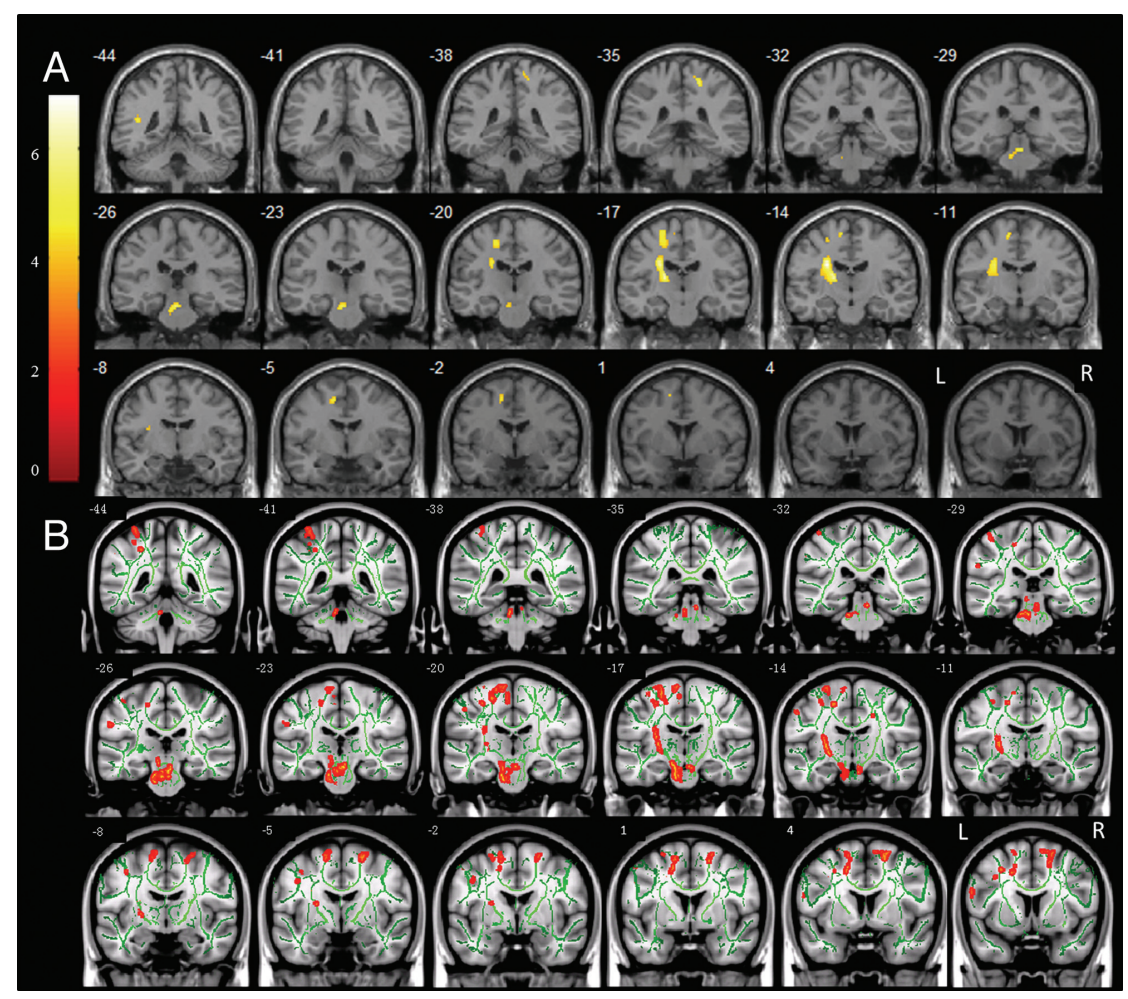

FIG 2. Significantly decreased FA was detected by VBM analysis $(A)$ and TBSS analysis $(B)$ in $C P H$ compared with PPH. The threshold was set at a combined cutoff value of $P<.001$ and a minimal cluster size of 20 voxels (for VBM analysis) or $P<.001$ (for TBSS analysis). Color scale $=t$ values; $\mathrm{L}=$ left; $\mathrm{R}=$ right. Arabic numeral indicates the location of each coronal section. Lesions were located on the left side.

Table 2: VBM analysis of FA maps between the CPH group and the PPH group

\begin{tabular}{|c|c|c|c|c|c|c|}
\hline \multirow[b]{2}{*}{ Regions } & \multicolumn{3}{|c|}{ MNI Coordinates } & \multirow[b]{2}{*}{ Peak $t$ Score } & \multirow[b]{2}{*}{ Number of Voxels } & \multirow[b]{2}{*}{ Volume (mL) } \\
\hline & $\mathbf{x}$ & y & z & & & \\
\hline Brain stem (IL) & -2 & -25 & -21 & 5.53 & 220 & 660 \\
\hline Sublobar (internal capsule/basal ganglia/thalamus) (IL) & -25 & -14 & 27 & 6.95 & 556 & 1668 \\
\hline Superior temporal gyrus (IL) & -42 & -45 & 15 & 4.81 & 68 & 204 \\
\hline Precentral gyrus (IL) & -20 & -18 & 54 & 4.92 & 122 & 366 \\
\hline Medial frontal gyrus (cingulate gyrus) (IL) & -13 & -4 & 51 & 5.05 & 62 & 186 \\
\hline Supplementary motor area (IL) & -11 & -12 & 60 & 5.23 & 54 & 162 \\
\hline Postcentral gyrus (CL) & 18 & -36 & 57 & 5.67 & 55 & 165 \\
\hline
\end{tabular}

Note:-The threshold was set at a combined cutoff value of $P<.001$ (uncorrected) and a minimal cluster size of 20 voxels. IL indicates ipsilesional; $\mathrm{CL}$, contralesional; MNI, Montreal Neurological Institute.

sional areas, brain stem, and contralesional cortical sensorimotor areas.

\section{ROI Analysis of FA Maps}

Compared with PPH, FA was decreased in CPH in the ROIs in the ipsilesional brain stem $(P<.01)$, medial frontal gyrus $(P<$ $.01)$, precentral gyrus $(P<.01)$, superior temporal gyrus $(P<$ $.01)$, supplementary motor area $(P<.01)$, sublobar areas $(P<.01)$, and contralesional postcentral gyrus $(P<.01)$.

Compared with healthy controls, FA was decreased in $\mathrm{CPH}$ in the ROIs in the ipsilesional brain stem $(P<.01)$, medial frontal gyrus $(P<.01)$, precentral gyrus $(P<.01)$, superior temporal gyrus $(P<.01)$, supplementary motor area $(P<.01)$, sublobar areas $(P<.01)$, and contralesional postcentral gyrus $(P<.01)$.

In a similar fashion, FA was decreased in PPH in the ROIs in the ipsilesional brain stem $(P<.05)$, medial frontal gyrus $(P<$ $.05)$, precentral gyrus $(P<.01)$, superior temporal gyrus $(P>$ $.05)$, supplementary motor area $(P<.05)$, sublobar areas
$(P<.01)$, and contralesional postcentral gyrus $(P<.05)$ compared with healthy controls (On-line Fig 2).

In addition, FA value correlated with Fugl-Meyer Assessment scores (hand+wrist) across all patients in the identified ROIs in the ipsilesional brain stem $(r=0.83 ; P<.01)$, medial frontal gyrus $(r=0.79 ; P<.01)$, precentral gyrus $(r=0.84 ; P<.01)$, superior temporal gyrus $(r=0.79 ; P<.01)$, supplementary motor area $(r=0.69 ; P<.01)$, sublobar areas $(r=0.90 ; P<.01)$, and contralesional postcentral gyrus $(r=0.80 ; P<.01$; On-line Fig 3$)$.

\section{DISCUSSION}

To the best of our knowledge, this study is the first that combines VBM and TBSS to investigate secondary degeneration in subgroups of patients who have had a stroke with different outcomes in hand function, and the differences in FA maps between $\mathrm{CPH}$ and PPH detected by the 2 methods were extremely consistent. Moreover, further quantitative analysis based on ROIs detected 
by group differences in our VBM analysis demonstrated that secondary degeneration arose in widespread regions of normal-appearing white matter after stroke, and the degree of degeneration correlated with outcomes in hand function.

Secondary degeneration of the pyramidal tract after focal motor pathway stroke has been reported in previous studies with use of DTI. At early or chronic stages, there is a reduction in FA value on the affected side in the fiber tract distal to the primary lesion. Moreover, the early decrease of FA correlates positively with motor deficits, and the more pronounced the degeneration of the pyramidal tract, the higher the motor deficit. ${ }^{13,14}$ A strong correlation between degeneration in the cerebral peduncle and functional outcome of the upper limbs has been detected in patients with chronic stage stroke. ${ }^{1}$ In addition, longitudinal studies have demonstrated that a progressive decrease of FA occurs along the pyramidal tract in the cerebral peduncle below the primary lesion. ${ }^{2,3}$ In accordance with previous studies, we found that FA of the ipsilesional brain stem significantly decreased in both $\mathrm{CPH}$ and PPH compared with healthy controls, and FA also significantly decreased in CPH compared with PPH. Furthermore, FA correlated positively with Fugl-Meyer Assessment scores (hand+wrist) across all patients. Our results not only demonstrate descending degeneration after focal motor pathway stroke but also further suggest that the degree of secondary degeneration in the ipsilesional brain stem contributes to different outcomes in hand function.

In addition, FA decreased in widespread regions of the motor system above the primary lesion in both $\mathrm{CPH}$ and $\mathrm{PPH}$ compared with healthy controls, including the ipsilesional medial frontal gyrus, precentral gyrus, superior temporal gyrus, supplementary motor area, and contralesional postcentral gyrus. A case of pontine infarction with retrograde degeneration has been detected and confirmed postmortem, ${ }^{15}$ and the progressive reduction of FA value in the fiber tract above the initial infarction also reflects retrograde degeneration proximal to the primary lesion. ${ }^{2}$ Two types of fiber tracts have been proposed in previous studies: 1) one is from neurons in the motor cortex, descending from the subcortex to the spinal cord and production pyramidal tract; and 2) the other is derived from relay nuclei in the thalamus that consist of basal ganglia-thalamocortical circuits. ${ }^{2,16-18}$ Moreover, Alexander et al (1986) ${ }^{16}$ suggested that the 5 basal ganglia-thalamocortical circuits are organized in parallel and that each circuit engages specific regions of the cerebral cortex involving the motor cortex, somatosensory cortex, supplementary motor area, cingulate motor cortex, and superior temporal gyrus. It is possible that decreased FA in widespread regions of the motor system above the primary lesion may be considered retrograde degeneration of different tract pathways, which leads to motor deficits after stroke.

We especially found decreased FA in the contralesional pyramidal tract near the sensorimotor cortex in both $\mathrm{CPH}$ and $\mathrm{PPH}$ compared with healthy controls. Although previous studies have reported secondary degeneration along the pyramidal tract on the affected side, often incorporating FA value in the contralesional regions as controls, ${ }^{3,19}$ the plasticity of the contralesional pyramidal tract has rarely been studied. One previous study reported that the FA of the normal-appearing white matter of the whole brain increased within 2 years after stroke, ${ }^{20}$ but it did not demonstrate how FA evolved in the contralesional white matter. More recently, a longitudinal study has demonstrated that a segment of the corticospinal tract (from the uppermost section of the cerebral peduncle to the lowest section of the pons) in the unaffected side did not have obvious plasticity. ${ }^{5}$ In line with these findings, we found no secondary degeneration on the unaffected side that correlated with outcomes in hand function, except the uppermost region of the pyramidal tract. It is important to note that a longitudinal study using resting-state functional MR imaging reported decreased functional connectivity of the bilateral primary sensorimotor cortex after stroke, nevertheless, in which the patients had different degrees of motor recovery. ${ }^{21}$ In contrast, our study, which investigated subgroups of patients with stroke, found decreased functional connectivity of the bilateral primary sensorimotor cortex in $\mathrm{CPH}$ compared with healthy controls; however, this finding was not observed in PPH compared with healthy controls (unpublished data). Therefore, we speculate that secondary degeneration of the contralesional pyramidal tract near the sensorimotor cortex is associated with functional reorganization of the bilateral motor cortex and that the degree of the degeneration may affect outcomes in hand function.

Our results not only show decreased FA in these identified ROIs in CPH compared with PPH, but they also exhibit a significantly positive correlation between FA value and Fugl-Meyer Assessment scores (hand+wrist). Recovery of motor function after stroke may occur for weeks or months and is attributed to cerebral reorganization. ${ }^{9,22}$ The impaired functional integrity of the corticospinal system may recruit secondary motor networks in both hemispheres to generate motor output to spinal cord motor neurons, but the secondary motor networks are less efficient at generating motor output, so this type of reorganization can be only partly successful in reducing motor deficits after stroke. ${ }^{10}$ Therefore, the outcome of motor function is likely to be attributed to the degree of damage of the primary motor system. Our findings suggest that the degree of secondary degeneration above the primary lesion correlates with the outcomes in hand function.

Our study did had have some open limitations in methodology. For example, ambiguity remains as to whether apparent differences highlighted by VBM approaches are attributed to differences in brain regions or local misalignment. ${ }^{23}$ Smoothing can be used to partly reduce intersubject variability and misalignments, but it simultaneously increases partial volume effects. TBSS does not require smoothing and performs alignment across participants effectively. However, processing FA maps with use of TBSS is easily affected by white matter degeneration caused by neurologic diseases. In addition, the spatial accuracy of TBSS analysis is limited by the skeleton and, therefore, does not provide significant detail in some locations. Moreover, it compares extremely different skeletons to replace the complete brain white matter structure. ${ }^{24}$ To confirm the accuracy and validity of our findings, we combined VBM and TBSS as complementary methods. In addition, we adopted an optimized strategy for registering FA maps by using individual $b=0$ images, and we carefully examined the result of normalization for each participant. Better methods and algorithms for voxel-based analysis across the whole brain are desired to further validate the reliability of our results. Finally, we 
did not consider the extent of leukoaraiosis, which will be involved in a future study.

\section{CONCLUSIONS}

Different outcomes in hand function in different subgroups of patients with stroke may be attributed to different degrees of secondary degeneration in widespread regions of the motor system. Therefore, our findings of secondary degeneration in these regions can serve as a potential biomarker for outcomes in hand function after subcortical stroke.

\section{ACKNOWLEDGMENTS}

We thank all of the volunteers and patients for their participation in this study. We also thank the Shanghai Key Laboratory of Magnetic Resonance, East China Normal University, for materials and technical support.

\section{REFERENCES}

1. Lindberg PG, Skejø PH, Rounis E, et al. Wallerian degeneration of the corticofugal tracts in chronic stroke: a pilot study relating diffusion tensor imaging, transcranial magnetic stimulation, and hand function. Neurorehabil Neural Repair 2007;21:551-60

2. Liang Z, Zeng J, Liu S, et al. A prospective study of secondary degeneration following subcortical infarction using diffusion tensor imaging. J Neurol Neurosurg Psychiatry 2007;78:581-86

3. Thomalla G, Glauche V, Weiller C, et al. Time course of Wallerian degeneration after ischaemic stroke revealed by diffusion tensor imaging. J Neurol Neurosurg Psychiatry 2005;76:266-68

4. Giuliani NR, Calhoun VD, Pearlson GD, et al. Voxel-based morphometry versus region of interest: a comparison of two methods for analyzing gray matter differences in schizophrenia. Schizophr Res 2005;74:135-47

5. Yu C, Zhu C, Zhang Y, et al. A longitudinal diffusion tensor imaging study on Wallerian degeneration of corticospinal tract after motor pathway stroke. Neuroimage 2009;47:451-58

6. Ashburner J, Friston KJ. Voxel-based morphometry-the methods. Neuroimage 2000;11:805-21

7. Smith SM, Jenkinson M, Johansen-Berg H, et al. Tract-based spatial statistics: voxelwise analysis of multi-subject diffusion data. Neuroimage 2006;31:1487-505

8. Afzali M, Soltanian-Zadeh H, Elisevich KV. Tract based spatial statistical analysis and voxel based morphometry of diffusion indices in temporal lobe epilepsy. Comput Biol Med 2011;41:1082-91

9. Ward NS, Brown MM, Thompson AJ, et al. Neural correlates of motor recovery after stroke: a longitudinal fMRI study. Brain 2003; 126:2476-96

10. Ward NS, Newton JM, Swayne OB, et al. Motor system activation after subcortical stroke depends on corticospinal system integrity. Brain 2006;129:809-19

11. Folstein MF, Folstein SE, McHugh PR. "Mini-mental state". A practical method for grading the cognitive state of patients for the clinician J Psychiatr Res 1975;12:189-98

12. Fugl-Meyer AR, Jääskö L, Leyman I, et al. The post-stroke hemiplegic patient. 1. a method for evaluation of physical performance. Scand J Rehabil Med 1975;7:13-31

13. Thomalla G, Glauche V, Koch MA, et al. Diffusion tensor imaging detects early Wallerian degeneration of the pyramidal tract after ischemic stroke. Neuroimage 2004;22:1767-74

14. Puig J, Pedraza S, Blasco G, et al. Wallerian degeneration in the corticospinal tract evaluated by diffusion tensor imaging correlates with motor deficit $\mathbf{3 0}$ days after middle cerebral artery ischemic stroke. AJNR Am J Neuroradiol 2010;31:1324-30

15. Kobayashi S, Hasegawa S, Maki T, et al. Retrograde degeneration of the corticospinal tract associated with pontine infarction. J Neurol Sci 2005;236:91-93

16. Alexander GE, DeLong MR, Strick PL. Parallel organization of functionally segregated circuits linking basal ganglia and cortex. Annu Rev Neurosci 1986;9:357-81

17. Parent A, Hazrati LN. Functional anatomy of the basal ganglia. I. The cortico-basal ganglia-thalamo-cortical loop. Brain Res Brain Res Rev 1995;20:91-127

18. McFarland NR, Haber SN. Convergent inputs from thalamic motor nuclei and frontal cortical areas to the dorsal striatum in the primate. J Neurosci 2000;20:3798-813

19. Pierpaoli C, Barnett A, Pajevic S, et al. Water diffusion changes in Wallerian degeneration and their dependence on white matter architecture. Neuroimage 2001;13:1174-85

20. Wang C, Stebbins GT, Nyenhuis DL, et al. Longitudinal changes in white matter following ischemic stroke: a three-year follow-up study. Neurobiol Aging 2006;27:1827-33

21. Park $\mathrm{CH}$, Chang WH, Ohn SH, et al. Longitudinal changes of resting-state functional connectivity during motor recovery after stroke. Stroke 2011;42:1357-62

22. Calautti C, Baron JC. Functional neuroimaging studies of motor recovery after stroke in adults: a review. Stroke 2003;34:1553-66

23. Anjari M, Srinivasan L, Allsop JM, et al. Diffusion tensor imaging with tract-based spatial statistics reveals local white matter abnormalities in preterm infants. Neuroimage 2007;35:1021-27

24. El-Rafei A, Engelhorn T, Wärntges S, et al. A framework for voxelbased morphometric analysis of the optic radiation using diffusion tensor imaging in glaucoma. Magn Reson Imaging 2011;29:1076-87 